\title{
Evaluación económica del tratamiento de artritis reumatoide con terapia biológica anti TNF en Colombia
}

\author{
Economic evaluation of the treatment of \\ rheumatoid arthritis with anti-TNF biological \\ therapy in Colombia
}

\author{
Gerardo Quintana • Bogotá, D.C., Juan Pablo Restrepo • Armenia, \\ Heidy Cáceres, Juan David Rueda, Diego Rosselli • Bogotá, D.C.
}

\section{Resumen}

Introducción: la artritis reumatoide (AR) es una enfermedad crónica de alto costo para el sistema de salud, en particular cuando se requiere terapia biológica.

Objetivos: diseñar un modelo económico para la toma de decisiones entre adalimumab (ADA), etanercept (ETA) e infliximab (INF) en el contexto colombiano.

Metodología: se diseñó un modelo de Markov, con un horizonte temporal de dos años, una perspectiva de un tercero pagador, que midiera efectividad clínica (en proporción de pacientes con respuesta ACR50 o mayor), abandono de terapia, eventos adversos, calidad de vida en AVAC (años de vida ajustados por calidad) y costos directos de tratamiento en pesos colombianos.

Resultados: la suspensión de la terapia fue mayor para INF y menor para ETA. La ganancia en AVAC fue ligeramente más alta para ETA y para ADA que para INF. Los costos anuales promedio de la terapia con INF fueron \$44.8 millones, para ADA \$41.0 millones, y \$39.0 millones para ETA. El costo promedio por mes logrado en ACR50 o superior fue de $\$ 9.37$; $\$ 8.83$ y $\$ 13.5$ millones, respectivamente, para ADA, ETA e INF.

Conclusiones: dadas las limitaciones y los supuestos del modelo, se podría concluir que, en el paciente colombiano "caso tipo" con AR, ETA es dominante sobre ADA e INF al tener un costo global total menor, y una efectividad superior a INF y por lo menos igual a la de ADA (Acta Med Colomb 2011; 36: 24-29).

Palabras clave: artritis reumatoide, terapia biológica, análisis económico, costo-utilidad.

\section{Abstract}

Introduction: rheumatoid arthritis (RA) is a chronic illness that implies high direct and indirect costs for the health system, in particular when biological therapy is prescribed.

Objectives: the aim of this study was to design an economic model for decision-making between adalimumab (ADA), etanercept (ETA) and infliximab (INF) in the Colombian context.

Methods: we designed a Markov model with a time horizon of two years and a third party payer perspective, measuring the effectiveness (as proportion of patients with ACR50 response or better) discontinuation of therapy, adverse events, quality of life in QALY (quality adjusted life years) and direct cost represented in Colombian pesos (1 US\$ = Col\$1800 pesos, aprox).

Results: discontinuation of therapy was highest for INF, and lowest for ETA. The gain in QALYs was somewhat higher for ETA and ADA, compared with INF. The total annual costs of the therapy with INF was $\$ 44.8$ million, $\$ 41.0$ million for ADA, and $\$ 39.0$ for ETA. The cost per month with ACR50 or higher was $\$ 9.37$; $\$ 8.83$ and $\$ 13.5$ million, respectively for ADA, ETA and INF.

Conclusion: given the limitations and given the assumptions of this model, we conclude that in the average Colombian patient with AR, ETA is dominant over ADA and INF, by having a lower total cost and at least the same effectiveness as ADA and higher than INF (Acta Med Colomb 2011; 36: 24-29).

Key words: rheumatoid arthritis, biological therapy, economic analysis, QALY, cost-utility
Dr. Gerardo Quintana: MSc, Internista Reumatólogo, Profesor Universidad Nacional de Colombia, Profesor Universidad de los Andes, Bogotá, D.C.; Dr. Juan Pablo Restrepo: Reumatólogo, Profesor Universidad del Quindío, Armenia; Dra. Heidy Cáceres: MSc, MHE: Magistra en Economía de la Salud, Pfizer S.A.; Dr. Juan David Rueda: Médico, Departamento de Epidemiología Clínica y Bioestadística, Universidad Javeriana; Dr. Diego Rosselli: EdM, MSc, Médico Neurólogo, Profesor Departamento de Epidemiología Clínica y Bioestadística, Universidad Javeriana, Bogotá, D.C

Este trabajo recibió el apoyo económico de Pfizer Colombia.

Correspondencia. Dr. Diego Rosselli, Departamento de Epidemiología Clínica y Bioestadística, Universidad Javeriana, Bogotá, D.C., Colombia

E-mail: diego.rosselli@gmail.com Recibido: 18/I/2011 Aceptado: 01/II/2011 


\section{Introducción}

La artritis reumatoide (AR) es una enfermedad crónica con gran morbilidad asociada, así como un alto impacto económico (1). La enfermedad representa un alto costo directo para el sistema de salud (entre los años 2003 y 2005 representó el sexto diagnóstico más frecuente y el quinto más costoso de los recobros totales del Fosyga, representando casi \$ 13.000.000.000) (2). Además, genera costos directos, indirectos e intangibles de un valor aún no calculado para la sociedad. Uno de los factores que ha incidido en el incremento de los costos directos para los pagadores del sistema es el empleo de la terapia biológica (TB) $(3,4)$, que está indicada en los pacientes que presentan pobre respuesta al manejo convencional con metotrexato, o contraindicación para su uso.

Las tres TB con antifactor de necrosis tumoral alfa más empleadas en Colombia son adalimumab (Humira $\left.{ }^{\circledR}\right)$, etanercept (Enbrel®) e infliximab (Remicade $®) ~(5)$. El objetivo de este estudio fue comparar, mediante un análisis de costoutilidad y costo-efectividad, y desde la perspectiva del sistema de salud colombiano, estas tres alternativas terapéuticas (que serán abreviadas ADA, ETA e INF, respectivamente).

Este modelo empleó información proveniente de registros de pacientes (equivalente a lo que se denomina en investigación estudios clínicos Fase IV). En pocas enfermedades se tiene la ventaja de emplear números grandes de sujetos que son seguidos en el "mundo real", y no en el ambiente artificial de los estudios clínicos, dado que el comportamiento de los medicamentos en la vida real es diferente al que se plantea en los grandes estudios aleatorios $(6,7)$. Para información de seguridad, así como de adherencia de los pacientes a los tratamientos -variables cruciales en este modelo-, los datos de los registros son más fiables que los de los estudios clínicos (8). Para efectividad, en cambio, nada supera a los estudios aleatorizados, ya que los registros pueden tener muchos sesgos. En el mundo real, por ejemplo, la asignación de un tratamiento en particular puede depender de muchos factores. Así, los médicos pueden prescribir los "mejores" tratamientos a los pacientes con la enfermedad más severa; una menor eficacia, en este caso, podría asociarse erróneamente con un medicamento que en realidad es más efectivo. Otro problema de los registros estriba en el orden de aparición de los medicamentos en un determinado mercado; el segundo medicamento en aparecer se empezará a prescribir en aquellos pacientes a quienes les falló el primer medicamento, y nuevamente una menor eficacia podría atribuirse erróneamente a un medicamento que es igual o superior en efectividad clínica.

Con estas salvedades en mente, en este modelo se emplearon las medidas de efectividad principalmente derivadas de estudios clínicos, y la seguridad y adherencia obtenidas de los registros internacionales de pacientes. Adicionalmente, se evaluó utilidad en años de vida ajustados por calidad (AVAC), derivados también de la literatura internacional.
A estos datos clínicos se les asignaron costos locales, en pesos colombianos.

\section{Material y métodos}

Para la obtención de la información se construyó de novo un modelo de Markov con ciclos trimestrales, diseñado desde la perspectiva de un tercero pagador (que para el caso colombiano sería el Sistema General de Seguridad Social en Salud, aplicable también a empresas promotora de salud -EPS- o a administradoras de regímenes especiales, por ejemplo las fuerzas militares). Se empleó un horizonte de tiempo de dos años (o sea ocho ciclos de Markov) para representar con más precisión las tasas de respuesta de la AR a la TB e incluir los principales eventos adversos potenciales de la medicación. Este horizonte temporal excluye algunos de los eventos adversos a mediano y largo plazo, pero contempla la respuesta clínica a la medicación.

\section{Estrategia de búsqueda}

El modelo se construyó empleando medidas de efectividad, seguridad y utilidad tomadas de la literatura internacional. Aunque para estimar la efectividad se incluyeron los estudios "pivotales" de cada una de las tres TB comparadas en el modelo (estudio ATTRACT para infliximab [9], estudio ARMADA para adalimumab [10] y el estudio TEMPO para etanercept [11]), las otras variables clínicas empleadas se obtuvieron principalmente de los registros de pacientes con AR.

Para localizar esta información en la literatura indexada se procedió de la siguiente manera: empleando el portal de búsqueda de Pubmed, se cruzó la palabra clave para AR ("rheumatoid arthritis"[mesh]) con los términos para cada una de las tres TB (adalimumab OR etanercept OR infliximab). Esta búsqueda arrojó un total de 2599 referencias (búsqueda actualizada hasta agosto 31 de 2010). Luego se aplicaron los siguiente límites a la búsqueda: Humans, Clinical Trial, All Adult: 19+ years, only items with abstracts. La búsqueda se redujo así a 381 referencias. Se revisaron los resúmenes de estas referencias buscando estudios que incluyeran por lo menos 50 pacientes con AR tratados con una o más de las TB de interés. Se localizaron así: 31 artículos cuyos textos completos fueron revisados. De las referencias bibliográficas de éstos se ubicaron siete artículos más, para un total de 38 artículos.

Al revisar estos documentos se encontraron datos duplicados, provenientes de un mismo registro, por lo que los datos empleados para la construcción del modelo se derivaron de 19 registros, provenientes de 12 países, con información de más de 30000 pacientes (Tabla 1).

\section{Características del modelo}

Se definieron cuatro diferentes estados de Markov (Figura 1): 1) el estado inicial, que lo llamamos "AR Primera Línea”, representa el paciente con AR que cumple con las indicaciones de la TB, y la recibe por primera vez. Después de uno o máximo dos ciclos de Markov [que equivalen a 
Tabla 1. Registros internacionales de pacientes con AR, empleados en el modelo.

\begin{tabular}{|lccc|}
\hline Referecia & Año & País & n \\
\hline Strangfeld (12) & 2009 & Alemania & 1796 \\
Barra (13) & 2009 & Canadá & 247 \\
Pérez (14) & 2008 & España & 442 \\
Bernal (15) & 2006 & España & 99 \\
Fernández (16) & 2007 & España & 161 \\
Ollendorf (17) & 2009 & Estados Unidos & 2382 \\
Yazici (18) & 2009 & Estados Unidos & 9074 \\
Tang (19) & 2008 & Estados Unidos & 1242 \\
Wu (20) & 2008 & Estados Unidos & 708 \\
Levälampi (21) & 2007 & Finlandia & 96 \\
Duclos (22) & 2006 & Francia & 770 \\
Flendrie (23) & 2003 & Holanda & 230 \\
Kievit (24) & 2003 & Holanda & 916 \\
Marchesoni (25) & 2009 & Italia & 1064 \\
Hyrich (26) & 2006 & Reino Unido & 7818 \\
Askling (27) & 2007 & 4167 \\
Kristensen (28) & 2008 & 1565 \\
Du Pan (29) & & 2364 \\
Inanc (30) & Suecia & 178 \\
n representa el número de pacientes de cada registro. & \\
\hline
\end{tabular}

máximo seis meses) (ver, por ejemplo,(11)] el paciente tiene la opción de responder con un ACR50 o más, en cuyo caso pasa al estado 2: "Responde ACR50".

La frecuencia de respuesta para cada una de las TB fue tomada según la tendencia global de los diferentes registros $(6,12,22,29,31)$. Una segunda opción que tiene el paciente de "AR Primera Línea" es responder parcialmente, sin alcanzar el ACR50 y pasando a un estado 3: que denominamos "Responde <ACR50". Finalmente, el estado 4 se denominó "Segunda Línea", y corresponde a un grupo heterogéneo de pacientes que por falta de efectividad o presentación de evento adverso, se cambian a otra de las tres TB consideradas en este estudio, reciben otro anti-TNF, o pasan a otros esquemas de medicación.

\section{Supuestos generales del modelo}

Efectividad: la principal medida de efectividad empleada fue el ACR50; aunque los estudios más recientes utilizan medidas más exigentes (como por ejemplo el ACR70), se escogió el ACR50 porque es el más comúnmente reportado en los registros. Las probabilidades de respuesta ACR50 en el segundo ciclo (o sea a los seis meses de iniciado el tratamiento) fueron $50 \%$ para ADA y para ETA, y $33 \%$ para INF.

Seguridad y abandono de medicación: los eventos adversos realizarán tres papeles importantes en este modelo: implican un costo, afectan la calidad de vida del paciente, y favorecen la suspensión de la medicación. Esta última

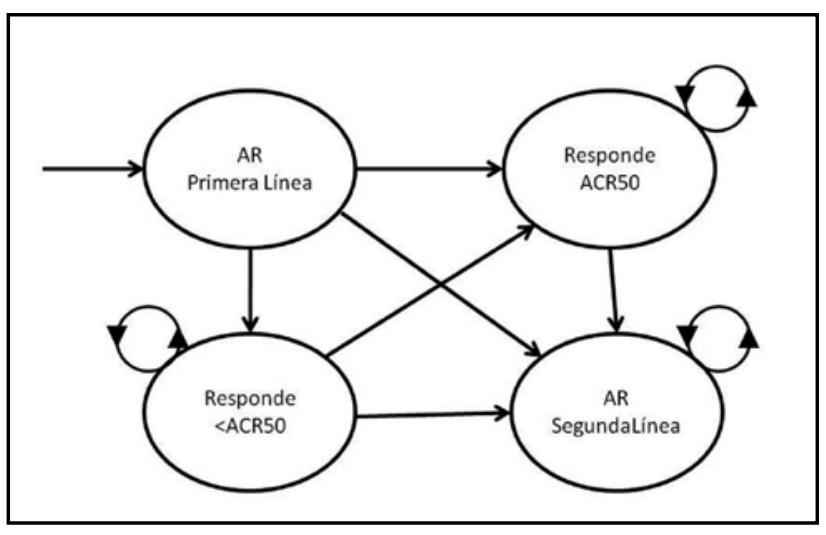

Figura 1. Esquema general del modelo.

variable -la suspensión- está bien cuantificada en los registros internacionales, particularmente en el registro alemán (12), en el suizo $(29,32)$ y en uno de los norteamericanos (17). A partir de estos registros se modelaron las tasas de suspensión de los tres medicamentos. Un supuesto del modelo es que la mitad de las suspensiones se deben a eventos adversos (la otra mitad a falta de efectividad). Este supuesto se infiere de los estudios de Du Pan en Suiza (29) y Marchesoni en Italia (25). A partir de esta información, se calcula cuál sería la probabilidad de eventos adversos serios para cada medicamento a lo largo del horizonte temporal del modelo. Los datos de probabilidad de eventos adversos de los estudios clínicos no se tuvieron en cuenta, ya que no son comparables por diferencias en definición de evento adverso, y sobre todo por tratarse de seguimientos relativamente cortos. Los eventos adversos graves reportados en los registros incluirían infecciones serias (como tuberculosis y neumonías), neoplasias, y complicaciones cardiovasculares serias (como infarto agudo de miocardio). En el modelo se asumió que los eventos adversos en los tres medicamentos fueron mayores en el primer trimestre (ADA 3.5\%, ETA $2.6 \%$, INF $4.4 \%$ ), con tendencia a reducirse de una manera similar para los tres a lo largo del tiempo.

En los registros internacionales la tasa de descontinuación de la medicación es mayor para INF, seguida de ADA y ETA, de estos últimos sólo uno reporta diferencia de $2 \%$ a favor de ADA, y los restantes muestran ventajas entre 5 y $10 \%$ para ETA. El estudio que muestra una mayor adherencia a INF es el trabajo de Tang et al. (19). Al analizar una base de datos de 82 HMO norteamericanas (equivalentes a nuestras EPS) buscando pacientes que recibían TB asociada a MTX (ADA 145 pacientes, ETA 607, INF 490) encontraron unas tasas de persistencia al año $70.8 \%$ para ADA, $72.8 \%$ para ETA, y $74.6 \%$ para INF. En el registro italiano (25), que incluye 1064 pacientes (ADA 303, ETA 242, INF 519), la tasa de suspensión a los 36 meses fue: ADA 46.4\% (48.4\% de ellos por EA), ETA 37.5\% (41.9\% por EA), INF $50.9 \%$ (54.5\% por EA). Algo similar ocurre en el registro británico, que incluye 6739 pacientes (26), 
y muestra tasas de descontinuación en primera línea: INF $42 \%$, ADA 30\%, ETA 29\%. Entre los suizos (29), a los dos años, 66\% de los pacientes continuaban con ADA, $65 \%$ con ETA y $58 \%$ con INF $(\mathrm{p}<0.001)$. Finalmente, en el registro holandés (23), al año, 73\% de los pacientes con ADA (n=94), 66\% de los de INF $(n=120)$ y $74 \%$ de los de ETA estaban con la medicación.

En general y para el horizonte temporal los parámetros correspondientes al modelo aparecen en la Tabla 2, como medidas acumuladas al final de los 24 meses (ocho ciclos).

Utilidad: en el modelo se emplearon utilidades en AVAC ( $Q A L Y$, por su sigla en inglés), tomadas de la literatura (adaptadas de 33). No se han estimado estos valores en población colombiana. Para los eventos adversos, se asumió una disutilidad equivalente a 0.39 AVAC aplicable tan sólo durante el trimestre en que este evento adverso se presente (una única vez). Las utilidades empleadas se muestran en la Tabla 3.

Costos: el modelo incorpora costos de medicamentos y costos de eventos adversos, únicamente. Se asume que los costos de consultas y de exámenes de laboratorio son similares en los diferentes grupos, y por tal razón es válido omitirlos.

Para los costos de medicamentos se emplearon las resoluciones del Ministerio de la Protección Social números 1265 de 2010 (para ADA e INF) y 574 de 2010 (para ETA), que fijaron los precios de recobro. Para el cálculo del costo se asumió la siguiente dosificación:

\begin{tabular}{lr} 
& Costo / mes \\
\hline ADA 40 mg c/2 semanas & $\$ 3.420 .000$ \\
ETA $50 \mathrm{mg}$ c/semana & $\$ 3.230 .000$ \\
INF 3mg x kg c/8 semanas (se asumió un paciente de $70 \mathrm{~kg}$ ) & $\$ 2.827 .000$ \\
\hline
\end{tabular}

Para el INF se asumió una aplicación inicial, seguida de dos aplicaciones en las semanas dos y seis, para posteriormente continuar con el esquema bimensual.

Adicionalmente para el INF se tomó en cuenta el ajuste de dosis reportado en el registro de Finckh (32), donde después de un año de tratamiento con INF se debe incrementar la dosis entre el $12 \%$ y el $18 \%$ bimensual. Sin embargo, tomando la opinión de los expertos en Colombia, este ajuste se hizo después del primer año de tratamiento, y con un incremento del $6 \%$ de la dosis inicial ajustándolo en cada ciclo (tres meses).

Para estimar el costo de un evento adverso se asumió que la mayor parte de éstos son leves $(16,27,34)$, (se asumen leves el $80 \%$ ). Los costos de un evento adverso grave se estimaron en $\$ 5.000 .000$, y los de uno leve en $\$ 500.000$.

Para el segundo año, se aplicaron tasas anuales de descuento de $3 \%$ tanto para costos como para AVAC.

\section{Resultados}

La Figura 2 muestra la tasa de respuesta, expresada en proporción de pacientes que logran ACR50 o superior. Nó-
Tabla 2. Probabilidades acumuladas (a 24 meses) empleadas en el modelo.

\begin{tabular}{|lccc|}
\hline Probabilidad de: & ADA $(\boldsymbol{\%})$ & ETA $(\boldsymbol{\%})$ & INF $(\boldsymbol{\%})$ \\
\hline Evento adverso serio & 12.3 & 9.5 & 15.6 \\
Suspensión de medicación & 25.0 & 19.1 & 31.2 \\
\hline
\end{tabular}

Tabla 3. Utilidades en años de vida ajustados por calidad (QALY) para cada estado. Adaptado de (33).

\begin{tabular}{|lc|}
\hline Estado & Utilidad en QALY \\
\hline AR en Primera Línea & 0.615 \\
Respuesta ACR50 & 0.835 \\
Respuesta <ACR50 & 0.705 \\
Promedio Segunda Línea* & 0.700 \\
\hline $\begin{array}{l}\text { * Para calcular esta utilidad se asumió lo siguiente: } 25 \% \text { de los pacientes que empiezan } \\
\text { segunda línea no tienen respuesta, 75\% presentan respuesta de algún tipo y los desenlaces } \\
\text { se distribuyen de la siguiente forma: 6\% suspende por evento adverso, 23\% suspende } \\
\text { por falta de eficacia, 6\% suspende por otra razón y 65\% tienen respuesta de ACR 50 o } \\
\text { menor a ACR 50 (con una respuesta 1,2 veces mayor que en la primera línea). }\end{array}$ \\
\hline
\end{tabular}

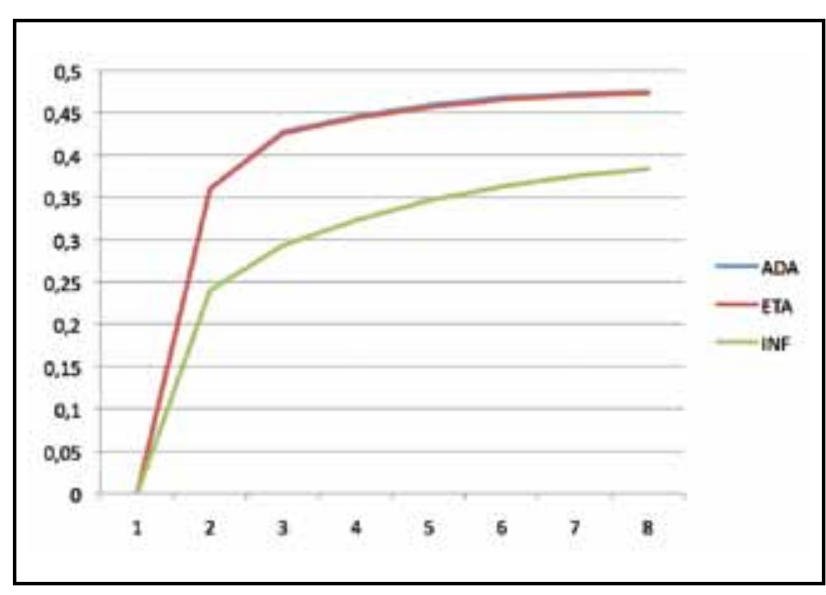

Figura 2. Respuesta a la medicación expresada en porcentaje de pacientes que llegan a ACR50. Para ADA y para ETA se asume efectividad similar, basados en un estudio de no inferioridad (15).

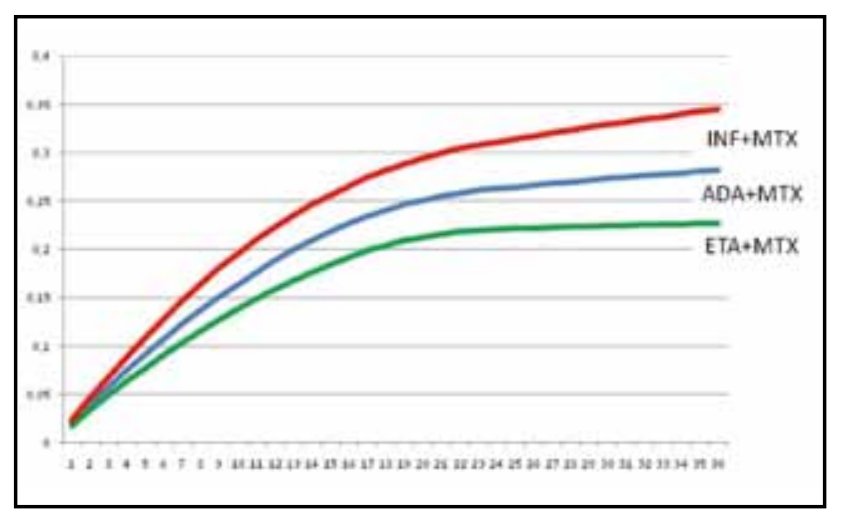

Figura 3. Gráfico de Kaplan-Meier generado por el modelo que ilustra el porcentaje de pacientes que ha suspendido medicación a lo largo del tiempo (en meses). En el modelo sólo se usaron los primeros 24 meses. 
tese que el modelo asume resultados similares para ADA y para ETA, basándose en el registro holandés $(23,24)$.

Al correr el modelo, se obtienen las tasas de abandono de la medicación que muestra la Figura 3. Estas tasas concuerdan con los resultados del registro alemán (12), como se muestra, para ADA, en la Figura 4 (curvas para ETA e INF no mostradas). El modelo concluye que hay un mayor abandono para INF, una mayor adherencia a ETA, y un resultado intermedio para ADA. La frecuencia de eventos adversos graves o que ameritan suspensión de medicación fue: para ADA 5.4\%, para ETA $4.1 \%$ y para INF $7.3 \%$.

Al estimar la calidad de vida, en AVAC, a lo largo de los dos años del horizonte temporal, se obtiene un valor de 1.440 para ADA, 1.443 para ETA y 1.411 para INF.

Los costos totales (y anuales promedio) en millones de pesos colombianos fueron $\$ 80.85$ (\$40.43) para ADA, $\$ 76.86$ (\$38.43) para ETA y, finalmente, \$88.41 (\$44.20) para INF. La proporción de este costo total que representan los eventos adversos es, en todos los casos, inferior al $1 \%$.

Con estos resultados, se podría calcular una razón neta de costo utilidad, en millones de pesos por AVAC, de $\$ 56.15$ para ADA, \$53.27 para ETA y \$62.65 para INF. Dado que sabemos cuántos pacientes logran el objetivo del ACR50 o más, y durante cuánto tiempo lo hacen, se puede estimar cuánto se paga por cada mes que el paciente logre esta meta de tratamiento. Este indicador de costo efectividad es: para ADA \$9.25 millones, para ETA \$8.72 millones y para INF $\$ 13.36$ millones por cada mes en ACR50 o superior.

Análisis de sensibilidad: dado que este modelo asume efectividad y seguridad similares para ADA y ETA (su diferencia en QALY es de 0.0029 QALY, lo que equivaldría en la práctica a un día adicional ajustado por calidad por cada dos año de tratamiento), el análisis de sensibilidad se convierte en un análisis de minimización de costos globales. Como el principal del costo en esta enfermedad es el medicamento (4), el equilibrio económico se logra cuando el costo mes de estos dos medicamentos, ADA y ETA, se iguala.

Al someter a análisis de sensibilidad al costo mes del INF,

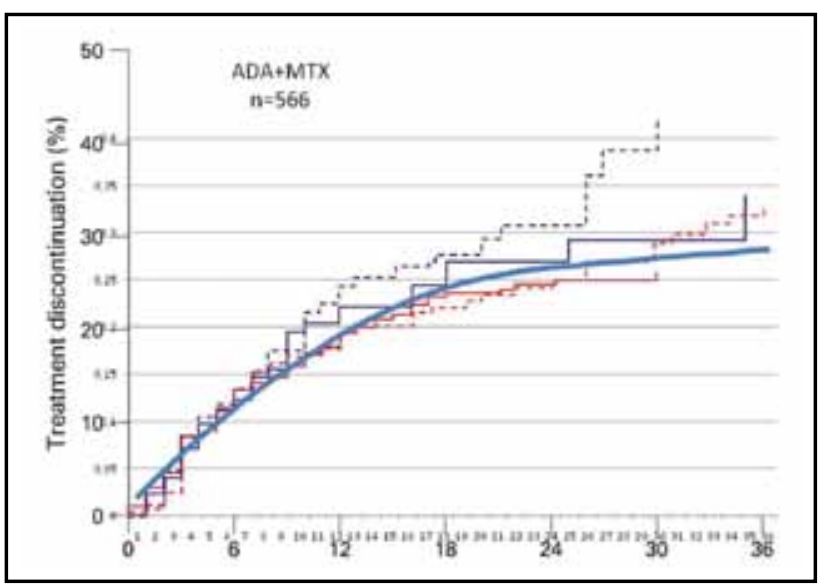

Figura 4. Sobreposición de la tasa de abandono de ADA en el modelo, graficada sobre el Kaplan Meier original del registro alemán (10). se encuentra que los costos globales de éste con el ADA se igualan si el INF costara \$2.55 millones por mes. En relación con el ETA, el costo del INF debería ser $\$ 2.25$ por mes. Vale recordar que ante costos globales iguales, se preferirían las otras dos alternativas terapéuticas sobre el INF, que son, así sea marginalmente, más efectivas y seguras.

Si el INF no requiriera ajuste de dosis, sería igual de costoso (globalmente) que el ADA, pero continuaría siendo más costoso que el ETA. El costo de los eventos adversos no es una variable crítica, y al modificarlo no se afecta el modelo.

\section{Discusión}

Todos los modelos económicos tienen limitaciones. Éste, en particular, ante la ausencia de datos locales, se basa en literatura foránea. Partimos de varios supuestos, basados en la práctica local y la opinión de expertos. Una realidad difícil de modelar es la variabilidad de la práctica, acrecentada por la ausencia de guías de práctica clínica. Algunos de los supuestos de este modelo podrían no ser aceptados de manera global, pero tienden a reflejar el buen juicio y criterio científico de las prácticas en Colombia.

Un dato que sí es inobjetable es el costo de la terapia, fijado en Colombia mediante norma gubernamental. Con esa base, se puede determinar que la opción menos costosa es el etanercept. Si su efectividad es equiparable a la del adalimumab, se podría concluir que también es la más costoefectiva. El infliximab no resulta muy favorecido en nuestro modelo, dado su costo, la necesidad de ajuste de dosis, la elevada deserción de los pacientes que lo toman y su perfil de seguridad, con mayor proporción de eventos adversos.

En conclusión, con estos resultados y aceptando las limitaciones y los supuestos de este modelo, se podría sugerir que, en el paciente tipo colombiano con artritis reumatoide, el etanercept es dominante sobre el infliximab al tener un costo global total menor, y una efectividad superior; de otro lado, es más costo-efectivo que el adalimumab, al tener un menor costo global y por lo menos igual efectividad.

\section{Referencias}

1. Klareskog L, Catrina AI, Paget S. Rheumatoid arthritis. Lancet 2009; 373: 659-72.

2. Cubillos L, Alfonso EA. Análisis descriptivo preliminar de los recobros en el Sistema General de Seguridad Social en Salud: 2002 a 2005. Bogotá: Ministerio de la Protección Social, 2005. Disponible en: http://mps.minproteccionsocial.gov. co/pars/library/documents/DocNewsNo16192DocumentNo4233.PDF (consultado febrero 23, 2011)

3. Mora C, Gonzalez A, Diaz J, Quintana G. Financial cost of early rheumatoid arthritis in the first year of medical attention: three clinical scenarios in a third-tier university hospital in Colombia. Biomedica 2009; 29: 43-50.

4. Pineda Tamayo R, Arcila GA, Restrepo P, Tobón GJ, Camargo JF,Anaya JM. Costos médicos directos de la artritis reumatoide. Rev Colomb Reumatol 2004; 11: 89-96.

5. Montoya N, Gómez L, Vélez M, Rosselli D. Análisis de costos del tratamiento de pacientes con artritis reumatoide en Medellín, Colombia. Rev Colomb Reumatol (en prensa)

6. Zink A, Strangfeld A, Schneider M, Herzer P, Hierse F, Stoyanova-Scholz M, et al. Effectiveness of tumor necrosis factor inhibitors in rheumatoid arthritis in an observational cohort study: comparison of patients according to their eligibility for major randomized clinical trials. Arthritis Rheum 2006; 54: 3399-407.

7. Yazici Y, Erkan D, Kulman I, Belostocki K, Harrison MJ. Decreased flares of 
rheumatoid arthritis during the first year of etanercept treatment: further evidence of clinical effectiveness in the "real world". Ann Rheum Dis 2002; 61: 638-40.

8. Zink A,Askling J, Dixon WG, Klareskog L, Silman AJ, Symmons DP. European biologicals registers: methodology, selected results and perspectives. Ann Rheum Dis 2009; 68: 1240-6.

9. Lipsky PE, van der Heijde DM, St Clair EW, Furst DE, Breedveld FC, Kalden JR, et al. Infliximab and methotrexate in the treatment of rheumatoid arthritis. $N$ Engl J Med 2000; 343: 1594-602.

10. Weinblatt ME, Keystone EC, Furst DE, Moreland LW, Weisman MH, Birbara CA, et al. Adalimumab, a fully human anti-tumor necrosis factor alpha monoclonal antibody, for the treatment of rheumatoid arthritis in patients taking concomitant methotrexate: the ARMADA trial. Arthritis Rheum 2003; 48: 35-45.

11. Klareskog L, van der Heijde D, de Jager JP, Gough A, Kalden J, Malaise $\mathbf{M}$, et al. Therapeutic effect of the combination of etanercept and methotrexate compared with each treatment alone in patients with rheumatoid arthritis: doubleblind randomised controlled trial. Lancet 2004; 363: 675-81.

12. Strangfeld A, Hierse F, Kekow J, von Hinueber U, Tony HP, Dockhorn R, et al. Comparative effectiveness of tumour necrosis factor alpha inhibitors in combination with either methotrexate or leflunomide. Ann Rheum Dis 2009; 68: 1856-62.

13. Barra L, Pope JE, Payne M. Real-world anti-tumor necrosis factor treatment in rheumatoid arthritis, psoriatic arthritis, and ankylosing spondylitis: costeffectiveness based on number needed to treat to improve health assessment questionnaire. J Rheumatol 2009; 36: 1421-8.

14. Perez B, Descalzo M, Carmona L. Reacciones adversas relacionadas con la administración de inhibidores del TNF: Análisis de un registro de terapias biológicas. Reumatol Clin 2008; 4: 90-5.

15. Bernal L, Guerrero A, Beltrán M, Hernández B, Colmenero MA. Efectividad y seguridad de adalimumab y etanercept en artritis reumatoide en un hospital de tercer nivel. Farm Hosp 2006; 30: 223-9.

16. Fernández-Nebro A, Irigoyen MV, Ureña I, Belmonte-López MA, Coret V, Jiménez-Núñez FG, et al.Effectiveness, predictive response factors, and safety of anti-tumor necrosis factor (TNF) therapies in anti-TNF-naive rheumatoid arthritis. J Rheumatol 2007; 34: 2334-42.

17. Ollendorf DA, Klingman D, Hazard E, Ray S. Differences in annual medication costs and rates of dosage increase between tumor necrosis factor-antagonist therapies for rheumatoid arthritis in a managed care population. Clin Ther 2009; 31: 825-35.

18. Yazici Y, Krasnokutsky S, Barnes JP, Hines PL, Wang J, Rosenblatt L. Changing patterns of tumor necrosis factor inhibitor use in 9074 patients with rheumatoid arthritis. J Rheumatol 2009; 36: 907-13.

19. Tang B, Rahman M, Waters HC, Callegari P. Treatment persistence with adalimumab, etanercept, or infliximab in combination with methotrexate and the effects on health care costs in patients with rheumatoid arthritis. Clin Ther 2008; 30: $1375-84$.

20. Wu E, Chen L, Birnbaum H, Yang E, Cifaldi M. Retrospective claims data analysis of dosage adjustment patterns of TNF antagonists among patients with rheumatoid arthritis. Curr Med Res Opin 2008; 24: 2229-40.
21. Levälampi T, Korpela M, Vuolteenaho K, Moilanen E. Etanercept and adalimumab treatment in patients with rheumatoid arthritis and spondyloarthropathies in clinical practice: adverse events and other reasons leading to discontinuation of the treatment. Rheumatol Int 2008; 28: 261-9.

22. Duclos M, Gossec L, Ruyssen-Witrand A, Salliot C, Luc M, Guignard S, Dougados M. Retention rates of tumor necrosis factor blockers in daily practice in 770 rheumatic patients. J Rheumatol 2006; 33: 2433-8.

23. Flendrie M, Creemers MC, Welsing PM, den Broeder AA, van Riel PL. Survival during treatment with tumour necrosis factor blocking agents in rheumatoid arthritis. Ann Rheum Dis 2003; 62: 30-3.

24. Kievit W, Adang EM, Fransen J, Kuper HH, van de Laar MA, Jansen TL, et al. The effectiveness and medication costs of three anti-tumour necrosis factor alpha agents in the treatment of rheumatoid arthritis from prospective clinical practice data. Ann Rheum Dis 2008; 67: 1229-34.

25. Marchesoni A, Zaccara E, Gorla R, Bazzani C, Sarzi-Puttini P, Atzeni F, et al. TNF-alpha antagonist survival rate in a cohort of rheumatoid arthritis patients observed under conditions of standard clinical practice. Ann N Y acad Sci 2009; 1173: $837-46$.

26. Hyrich KL, Lunt M, Watson KD, Symmons DP, Silman AJ. British Society for Rheumatology Biologics Register. Outcomes after switching from one anti-tumor necrosis factor alpha agent to a second anti-tumor necrosis factor alpha agent in patients with rheumatoid arthritis: results from a large UK national cohort study. Arthritis Rheum 2007; 56: 13-20.

27. Askling J, Fored CM, Brandt L, Baecklund E, Bertilsson L, Feltelius N. Time-dependent increase in risk of hospitalisation with infection among Swedish RA patients treated with TNF antagonists. Ann Rheum Dis 2007; 66: 1339-44.

28. Kristensen LE, Kapetanovic MC, Gülfe A, Söderlin M, Saxne T, Geborek P. Predictors of response to anti-TNF therapy according to ACR and EULAR criteria in patients with established RA: results from the South Swedish Arthritis Treatment Group Register. Rheumatology (Oxford) 2008; 47: 495-9.

29. Du Pan SM, Dehler S, Ciurea A, Ziswiler HR, Gabay C, Finckh A. Comparison of drug retention rates and causes of drug discontinuation between anti-tumor necrosis factor agents in rheumatoid arthritis. Arthritis Rheum 2009; 61: 560-8.

30. Inanc N, Direskeneli H. Serious infections under treatment with TNF-alpha antagonists compared to traditional DMARDs in patients with rheumatoid arthritis. Rheumatol Int 2006; 27: 67-71.

31. Perera LC, Tymms KE, Wilson BJ, Shadbolt B, Brook AS, Dorai Raj AK, et al. Etanercept in severe active rheumatoid arthritis: first Australian experience. Intern Med J 2006; 36: 625-31.

32. Finckh A, Simard JF, Gabay C, Guerne PA; SCQM physicians. Evidence for differential acquired drug resistance to anti-tumour necrosis factor agents in rheumatoid arthritis. Ann Rheum Dis 2006; 65: 746-52.

33. Chiou CF, Choi J, Reyes CM. Cost-effectiveness analysis of biological treatments for rheumatoid arthritis. Expert Rev Pharmacoecon Outcomes Res 2004; 4: $307-15$.

34. Bansback NJ, Brennan A, Ghatnekar O. Cost effectiveness of adalimumab in the treatment of patients with moderate to severe rheumatoid arthritis in Sweden. Ann Rheum Dis 2005; 64: 995-1002. 